\title{
The hepatitis B virus $X$ protein promotes tumor cell invasion by inducing membrane-type matrix metalloproteinase-1 and cyclooxygenase- 2 expression
}

\author{
Enrique Lara-Pezzi, ${ }^{1}$ Maria Victoria Gómez-Gaviro, ${ }^{2}$ Beatriz G. Gálvez, ${ }^{2}$ Emilia Mira, ${ }^{3}$ \\ Miguel A. Iñiguez, ${ }^{4}$ Manuel Fresno, ${ }^{4}$ Carlos Martínez-A., ${ }^{3}$ Alicia G. Arroyo, ${ }^{2}$ \\ and Manuel López-Cabrera ${ }^{1}$ \\ ${ }^{1}$ Unidad de Biología Molecular, and \\ ${ }^{2}$ Servicio de Inmunología, Hospital Universitario de la Princesa, Madrid, Spain \\ ${ }^{3}$ Centro Nacional de Biotecnología, and \\ ${ }^{4}$ Centro de Biología Molecular Severo Ochoa, Madrid, Spain
}

\begin{abstract}
Hepatocellular carcinoma is strongly associated with chronic infection by the hepatitis B virus (HBV) and has poor prognosis due to intrahepatic metastasis. $\mathrm{HBx}$ is often the only $\mathrm{HBV}$ protein detected in hepatic tumor cells; however, its contribution to tumor invasion and metastasis has not been established so far. In this work, we show that $\mathrm{HBx}$ enhances tumor cell invasion, both in vivo and in vitro. The increased invasive capacity induced by $\mathrm{HBx}$ is mediated by an upregulation of membrane-type 1 matrix metalloproteinase (MT1-MMP) expression, which in turn activates matrix metalloproteinase-2. Induction of both MT1-MMP expression and cell invasion by HBx is dependent on cyclooxygenase-2 (COX-2) activity. In addition, HBx upregulates the expression of COX-2, which is mediated by the transcriptional activation of the COX-2 gene promoter in a nuclear factor of activated $\mathrm{T}$ cell-dependent (NF-AT-dependent) manner. These results demonstrate the ability of $\mathrm{HBx}$ to promote tumor cell invasion by a mechanism involving the upregulation of MT1-MMP and COX-2 and provide new insights into the mechanism of action of this viral protein and its involvement in tumor metastasis and recurrence of hepatocellular carcinoma.
\end{abstract}

J. Clin. Invest. 110:1831-1838 (2002). doi:10.1172/JCI200215887.

\section{Introduction}

Hepatocellular carcinoma (HCC) is one of the most common malignancies worldwide and is frequently a terminal complication of chronic inflammatory and fibrotic liver disease (1). HCC treatment is palliative, and long-term survival is rare. Intrahepatic metastasis is a main cause of HCC recurrence and poor prognosis after tumor resection (1). Chronic hepatitis B virus (HBV) infection is strongly associated with the development of cirrhosis and HCC $(1$, 2). After integration of the HBV DNA into the host genome, the expression of the viral protein $\mathrm{HBx}$ becomes deregulated and is still found in transformed hepatocytes, even in the absence of any other viral marker $(2,3)$.

Received for publication May 8, 2002, and accepted in revised form October 29, 2002.

Address correspondence to: Manuel López-Cabrera, Unidad de Biología Molecular, Hospital Universitario de la Princesa, Diego de León 62, 28006 Madrid, Spain.

Phone: 34-91-5202334; Fax: 34-91-5202374;

E-mail:mlopez@hlpr.insalud.es.

Conflict of interest: The authors have declared that no conflict of interest exists.

Nonstandard abbreviations used: hepatocellular carcinoma (HCC); hepatitis B virus (HBV); matrix metalloproteinase (MMP); membrane-type 1 matrix metalloproteinase (MT1-MMP); cyclooxygenase (COX); nonsteroidal anti-inflammatory drug (NSAID); acetylsalicylic acid (ASA); chorioallantoic membrane (CAM); nuclear factor of activated T cell (NA-AT); prostaglandin $\mathrm{E}_{2}\left(\mathrm{PGE}_{2}\right)$.
Most efforts in the study of the role of HBx in HCC development have focused on its involvement in the genesis of liver carcinomas. In this regard, $\mathrm{HBx}$ is able to induce HCC either alone or in synergy with c-myc or chemical carcinogens in transgenic mice (4-6). HBx activates several signal transduction pathways that lead to the transcriptional upregulation of a number of cellular genes, including those of growth and angiogenic factors and oncogenes $(2,7)$. In addition, HBx promotes cell cycle progression, inactivates negative growth regulators like p53, and facilitates the accumulation of DNA mutations by interfering with the DNA repair machinery (2). HBx is also able to interfere with apoptotic signals, leading to tumor cell survival, although this issue remains controversial $(2,8)$. The possible role of $\mathrm{HBx}$ in the late stages of tumor progression and metastasis, however, has not been established.

The spread of a primary tumor to a secondary metastatic site requires cancer cells to be able to degrade the surrounding ECM and invade the lymphatic and blood vessels. Matrix metalloproteinases (MMPs) mediate ECM remodeling and are involved in a variety of pathophysiological processes, including tumor metastasis (9). Degradation of collagen IV, a main component of the basal lamina, is achieved by specific MMPs known as gelatinases (MMP-2 and MMP-9). MMP-2/gelatinase A is secreted as a proenzyme that is activated at the cell surface by membrane-type 1 matrix metalloproteinase 
(MT1-MMP) (9). Expression of MT1-MMP and activation of MMP-2 are associated with tumor invasion and metastasis and can be induced by different stimuli (9-11). Interestingly, it has recently been shown that expression of MT1-MMP and activation of MMP-2 can be regulated by cyclooxygenase-2 activity (12).

Cyclooxygenases (COXs) mediate the synthesis of prostaglandins from arachidonate (13). COX-1 shows a constitutive expression in different cell types and is involved in the homeostatic function of prostaglandins. In contrast, COX-2 expression can be induced by a number of mitogenic, inflammatory, and pro-oncogenic stimuli and plays a key role in a variety of processes, including the onset of the inflammatory response, mitogenesis, angiogenesis, and tumor progression and metastasis (13). In this context, COX-2 is expressed in several carcinomas, including HCC, and overexpression of this enzyme in transgenic mice is sufficient to induce tumorigenesis (13-15). In addition, a strong correlation has been established between the use of nonsteroidal anti-inflammatory drugs (NSAIDs) and a decreased incidence of different cancers (13).

In this study we describe the ability of HBx to induce tumor cell invasion, both in vivo and in vitro. This invasive behavior is dependent on MT1-MMP and COX-2 activity. Moreover, $\mathrm{HBx}$ upregulates the expression of COX-2, at both mRNA and protein levels. These results demonstrate the ability of this viral protein to induce tumor cell invasion and support a role for HBx in the late steps of tumor development and metastasis.

\section{Methods}

Cell lines, antibodies, and reagents. CMX and CMO cells are derivatives of Chang liver cells (CCL13; American Type Culture Collection, Manassas, Virginia, USA) and constitutively express low levels of $\mathrm{HBx}$ and the hygromycin resistance gene, respectively (16-18). The hepatic origin of Chang liver cells has been recently established by microarray assays (19). Three different CMX clones were used to rule out clone-specific results. 2.2.15 cells were derived from the human hepatoma cell line HepG2 by stably transfecting two head-to-tail copies of the HBV genome (20). HBx expression in 2.2.15 and CMX cells has been previously reported $(16,20) .4 \mathrm{pX}$ cells are stable transfectants, derived from the immortalized murine hepatocyte cell line AML-12, in which HBx expression can be induced by removing tetracycline for 24 hours (21). All the experiments were carried out in the absence of FCS. The blocking anti-MT1-MMP mAb's LEM 2/15 and LEM 1/58 were described previously (22). Anti-MMP-2 and anti-MMP-9 were purchased from R\&D Systems Inc. (Minneapolis, Minnesota, USA). Anti- $\alpha$-tubulin, HGF, acetylsalicylic acid (ASA), indomethacin, and the tetracycline analogue doxicycline were obtained from Sigma-Aldrich (St. Louis, Missouri, USA) and anti-albumin from DAKO A/S (Glostrup, Denmark). Anti-COX-2 and the COX-2 inhibitor NS398 were purchased from Alexis Corp. (San Diego, California, USA), and anti-COX-1 was from
Santa Cruz Biotechnology Inc. (Santa Cruz, California, USA). The MMP inhibitor BB-3103 was kindly provided by British Biotech (Oxford, United Kingdom). Meloxicam was a kind gift from Innogenetics $\mathrm{GmbH}$ (Heiden-Westfalen, Germany).

In vivo and in vitro invasion assays. In vivo invasion assays using chick embryos were performed as previously described (23). Briefly, 2 million cells were seeded onto the upper part of the chorioallantoic membrane (CAM) of a 9-day-old chick embryo and allowed to invade for 48 hours. Genomic DNA was extracted from the lower part of the CAM, and the presence of human Alu sequences was analyzed by quantitative $\mathrm{PCR}$.

For in vitro invasion assays of CMO, CMX, HepG2, and 2.2 .15 cells, $1 \mathrm{mg}$ of growth factor-reduced Matrigel (Becton Dickinson Immunocytometry Systems, Mountain View, California, USA) was added to the upper chamber of Transwells with $12-\mu \mathrm{m}$ pores and allowed to air-dry. After washing, $3 \times 10^{5}$ cells were seeded onto the upper chamber. For $4 \mathrm{pX}$ cells, $0.4 \mathrm{mg}$ of Matrigel was air-dried on $8-\mu \mathrm{m}$-pore Transwells, and $4 \times 10^{4}$ cells were seeded. Six hours later, $10 \mathrm{ng} / \mathrm{ml} \mathrm{HGF}$ was added to the lower chamber, and, where indicated, an inhibitor $(5 \mu \mathrm{M}$ BB-3103, $100 \mu \mathrm{M} \mathrm{NS} 398,60 \mu \mathrm{g} / \mathrm{ml}$ meloxicam, $60 \mu \mathrm{g} / \mathrm{ml}$ indomethacin, or $600 \mu \mathrm{g} / \mathrm{ml} \mathrm{ASA}$ ) or $20 \mu \mathrm{g} / \mathrm{ml} \mathrm{mAb}$ was added to both chambers. Cells were allowed to invade the Matrigel for 48 hours, after which the Transwells were fixed in $4 \%$ formaldehyde, the gel was removed with a cotton swab, and the filters were cut and stained with 4',6-diamidine-2'-phenylindole dihydrochloride (DAPI). The cells in eight different fields were counted using a fluorescence microscope. Each experiment was carried out in triplicate.

Western blot. A total of $3 \times 10^{5}$ cells were grown in the absence of FCS and, where indicated, treated with 10-100 $\mu \mathrm{M}$ NS398, $60 \mu \mathrm{g} / \mathrm{ml}$ meloxicam, $60 \mu \mathrm{g} / \mathrm{ml}$ indomethacin, or $600 \mu \mathrm{g} / \mathrm{ml}$ ASA. After 24 hours, cells were lysed in $60 \mu \mathrm{l}$ of Laemmli buffer, and both lysates and supernatants were analyzed by Western blot as described (18).

$R T$-PCR. Cells were grown for 24 hours in $0 \% \mathrm{FCS}$, RNA was extracted, and the cDNA was obtained from $1 \mu \mathrm{g}$ of total RNA by reverse transcription. Quantitative PCR of MT1-MMP and COX-2 was carried out in a LightCycler (Roche Diagnostics GmbH, Mannheim, Germany) using a SYBR Green kit (Roche Diagnostics $\mathrm{GmbH}$ ) and two specific primer sets (5'-AGGGGCGGTGAGCGCTGCTG-3' and $5^{\prime}$-TCAGACCTTGTCCAGCAGGG-3' for MT1-MMP; $5^{\prime}$-TTCAAATGAGATTGTGGGAAAATTGCT- $3^{\prime}$ and $5^{\prime}$-AGATCATCTCTGCCTGAGTATCTT- $3^{\prime}$ for COX-2).

Plasmids, transfections and luciferase assays. The reporter plasmids bearing different constructs of the human COX-2 promoter linked to the luciferase reporter gene have been previously described (24). pSV-X and the control vector pSV-hygro bear the $X$ gene and the hygromycin phosphotransferase gene, respectively, under the control of the SV40 early enhancer/promoter (25). The pSH102C $\Delta 418$ expression vector (a kind gift from Gerald Crabtree, Stanford University, Stanford, 

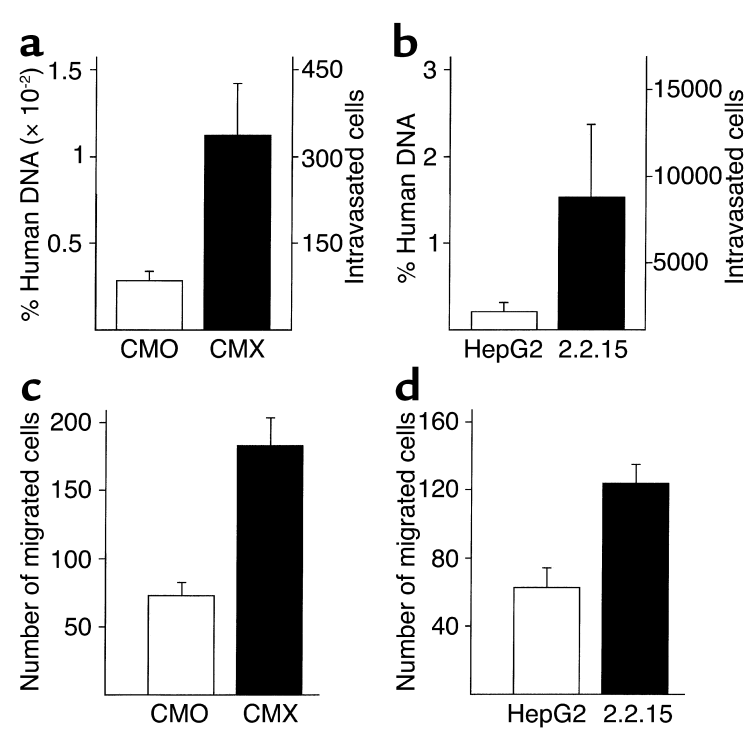

Figure 1

$\mathrm{HBx}$ induces tumor cell invasion in vivo and in vitro. (a and $\mathbf{b}$ ) In vivo invasion. $\mathrm{CMO}$ and $\mathrm{CMX}(\mathbf{a})$ or HepG2 and 2.2.15 (b) were analyzed for their ability to invade the CAM of a chick embryo. The results are expressed as percentage of human DNA (left) or as number of intravasated cells (right). Experiments were carried out at least in quadruplicate. (c and $\mathbf{d}$ ) In vitro invasion. The invasive capacity of CMO and CMX (c) or HepG2 and 2.2.15 (d) cells was tested using Matrigel-coated Transwells. Cells in the underside of the filter were stained, and eight independent fields were counted. The results are expressed as the mean value \pm SE of three independent points.

California, USA) derives from $\mathrm{pBJ} 5$ and encodes a nuclear factor of activated T cell-2 (NF-AT2) deletion mutant (1-418) that functions as a dominant negative for all NF-AT isoforms (25).

Transfections were carried out as previously reported (25), using $0.1 \mu \mathrm{g}$ of reporter plasmid, $4 \mu \mathrm{g}$ of pSV-X or pSV-hygro, and, where indicated, $1 \mu \mathrm{g}$ of pSH102C 4418 or $\mathrm{pBJ} 5$, using the DOSPER liposomal reagent (Roche Diagnostics $\mathrm{GmbH}$, Mannheim, Germany). After 6 hours, the medium was removed and cells were grown in DMEM with $2 \%$ FCS for 18 hours. Cells were lysed, and luciferase activity was measured.

\section{Figure 2}

$\mathrm{HBx}$-induced tumor cell invasion is dependent on metalloproteinase activity, and $\mathrm{HBx}$ upregulates activated MMP-2 expression. (a) CMO and CMX cells were allowed to invade a Matrigel-coated Transwell in the presence of the MMP inhibitor BB-3103 or DMSO as a control. The invasion was quantified as in Figure 1. (b) Cells were grown for 24 hours in serum-free medium, and gelatin zymography analysis of the cell culture supernatants of PMA-stimulated human umbilical vein endothelial cells (control) and the different hepatic cell lines was performed. (c and $\mathbf{d}$ ) CMO and $\mathrm{CMX}(\mathbf{c})$ or HepG2 and 2.2.15 (d) cell lysates and supernatants were analyzed for MMP-2 and MMP-9 expression by Western blot. Anti- $\alpha$-tubulin and anti-albumin mAb's assure equal protein load in all lanes.

\section{Results}

HBx induces tumor cell invasion. To determine whether HBx could induce tumor cell invasion, we used both in vivo and in vitro approaches. In vivo cell invasion was evaluated using a chick embryo invasion model in which cells are studied for their ability to degrade the embryo CAM and penetrate into the bloodstream (23). CMX cells displayed an invasive capacity fourfold higher than that of the control CMO cells (Figure 1a). In addition, 2.2.15 cells showed 7.9 times more invasive potential than their parental HepG2 cells (Figure 1b).

In vitro cell invasion was determined using a Matrigel invasion assay. CMX cells showed a 2.3-fold higher invasion efficiency than CMO cells (Figure 1c), and, similarly, 2.2 .15 cells showed increased invasive capacity with respect to HepG2 cells (Figure 1d), indicating that HBx was able to induce cell invasion both in vivo and in vitro.

$H B x$-induced invasion correlates with increased expression of activated MMP-2. To study the possible role of MMPs in $\mathrm{HBx}$-induced cell invasion, we first analyzed the effect of the general MMP inhibitor BB-3103 on the invasive capacity of CMX and CMO cells. Transwell invasion assays performed in the presence of BB-3103 showed a decreased number of both CMX and CMO cells in the bottom chamber of the Transwell (Figure 2a), suggesting the involvement of MMPs in the invasion of the gel by these cells.

To further support the relation between MMP expression and cell invasion, we tested the activity of different MMPs by zymography assays. As shown in Figure $2 b$, only a $66-\mathrm{kDa}$ band, likely corresponding to MMP-2, was detected by gelatin zymography in the culture supernatants of Chang liver and HepG2-derived cells. No MMP activity was detected when casein was used as substrate (data not shown), suggesting that no other MMPs were being secreted. We then analyzed the presence of the gelatinases MMP-2 and MMP-9 by Western blot both in the cell culture supernatants and in cell extracts. An increased amount of the activated $62-\mathrm{kDa}$ a

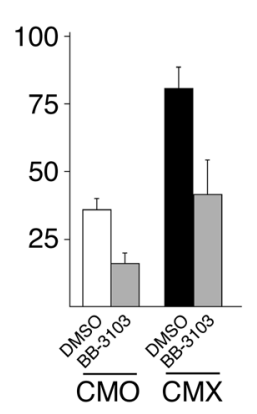

b

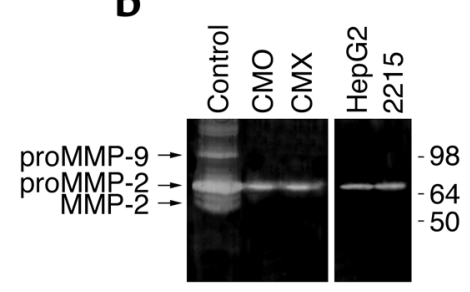

C
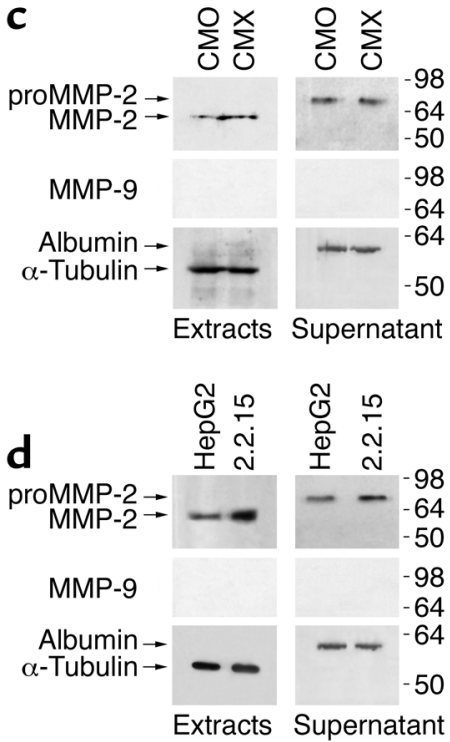
a

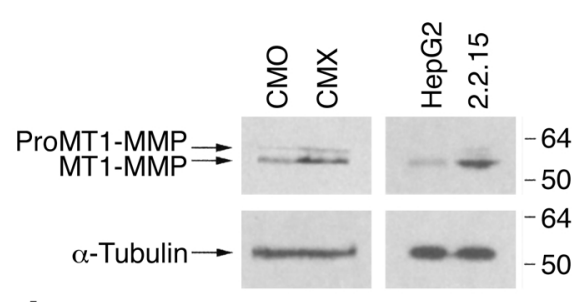

b

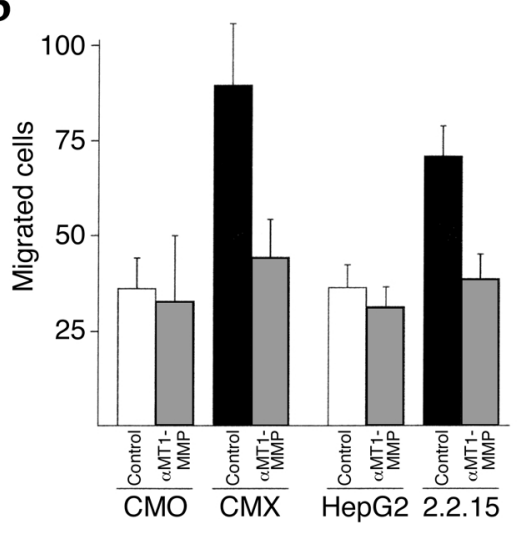

C

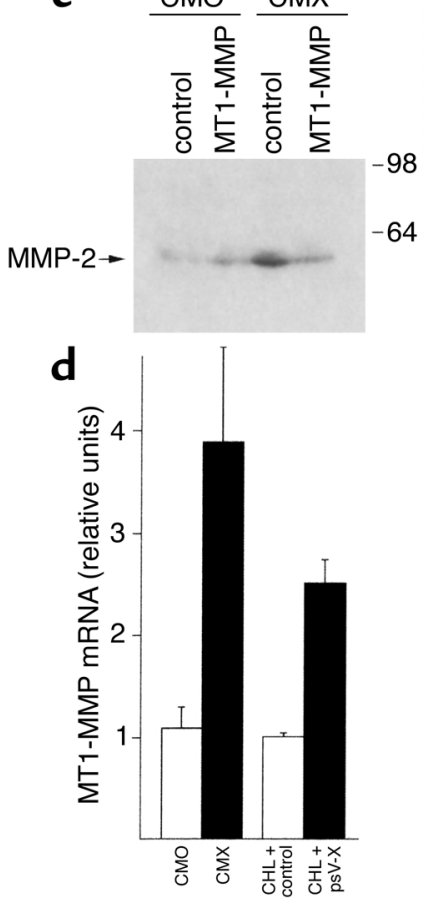

Figure 3

HBx-mediated increase of activated MMP-2 and cell invasion is due to the induction of MT1-MMP expression. (a) MT1-MMP expression was analyzed by Western blot in CMO, CMX, HepG2, and 2.2.15 cells grown for 24 hours in the absence of serum. (b) CMO, CMX, HepG2, or 2.2.15 cells were allowed to invade a Matrigel-coated Transwell in the presence of the anti-MT1-MMP mAb LEM 1/58 or a control antibody. The invasion was quantified as in Figure 1. (c) CMO and CMX cells were incubated for 24 hours in the presence of the anti-MT1-MMP blocking mAb LEM 1/58 or a control $\mathrm{mAb}$, and the presence of MMP-2 in the cell lysates was analyzed by Western blot. (d) The presence of MT1-MMP mRNA was studied by quantitative RT-PCR in CMO and CMX cells and in Chang liver cells (CHL) transiently transfected with an $\mathrm{HBx}$-expression vector or a control plasmid.

MMP-2 isoform was found in CMX cell extracts compared with CMO cells (Figure 2c), whereas the 66-kDa proMMP-2 isoform was not detected in the cell extracts (Figure 2c) unless the blot was overexposed (data not shown). On the contrary, the $66-\mathrm{kDa}$ form was the only MMP-2 isoform found in cell culture supernatants, although no significant differences in the expression of this protein were observed between CMX and CMO cells. Activated MMP-2 was also increased in 2.2.15 cell extracts compared with HepG2 extracts (Figure 2d). A variable amount of proMMP-2, which proved not to be significant, could be observed in the cell culture supernatants. MMP-9 was detected neither in the cell extracts nor in the cell culture supernatants (Figure 2, $c$ and d). It is noteworthy that when supernatants were concentrated more than fivefold, a small amount of proMMP-9 could be observed, and this amount was not different between CMX and CMO cells (our unpublished data).

Increase in activated MMP-2 in HBx-expressing cell extracts is dependent on MT1-MMP upregulation. To determine whether the activation of MMP- 2 correlated with an induction of MT1-MMP expression, we performed
Western blot studies. CMX cells showed increased expression of MT1-MMP when compared with CMO cells (Figure $3 a)$, correlating with the induction of activated MMP-2. Consistently, 2.2.15 cells also showed an increment in MT1-MMP expression compared with HepG2 cells (Figure 3a). To explore the involvement of MT1-MMP in the induction of cell invasion by $\mathrm{HBx}$, we tested the effect of the blocking anti-MT1MMP mAb LEM 1/58 (22) on the invasive capacity of the different cell lines. Blockade of the MT1-MMP catalytic site resulted in a loss of invasive capacity by CMX and 2.2.15 cells (Figure 3b). In addition, inhibition of MT1-MMP activity resulted in a decrease of activated MMP-2 in CMX cells (Figure 3c), as assayed by Western blot, suggesting that MT1-MMP played an important role in the induction of tumor cell invasion and MMP-2 activation by HBx. Accordingly, an upregulation of MT1-MMP mRNA was observed by quantitative RT-PCR both in CMX cells and in Chang liver cells transiently transfected with an $\mathrm{HBx}$ expression vector (Figure 3d).

HBx-induced MT1-MMP expression and tumor cell invasion are sensitive to COX-2 inbibitors. Next, we explored the effect of different COX-2 inhibitors on $\mathrm{HBX}$ induced cell invasion. The COX-2-specific inhibitor NS398 blocked the induction of MMP-2 activation by HBx in a dose-dependent manner, and this inhibition could be reversed by the addition of prostaglandin $\mathrm{E}_{2}\left(\mathrm{PGE}_{2}\right)$ (Figure $\left.4 \mathrm{a}\right)$. In addition, treatment of CMX and 2.2.15 cells with $100 \mu \mathrm{M}$ NS398 resulted in a decrease of MT1-MMP expression, whereas it had no effect on CMO or HepG2 cells (Figure 4b). Analysis of MT1-MMP mRNA expression by quantitative RT-PCR in CMX and CMO cells treated with 100 $\mu M$ NS398 showed a clear inhibition of MT1-MMP mRNA synthesis by CMX cells (Figure 4c), confirming a role for COX-2 in the induction of MT1-MMP expression by HBx. In contrast, little or no decrease in MMP-2 mRNA was observed in CMX or CMO cells (not shown). More interestingly, the invasive ability of CMX, but not CMO, cells was dramatically decreased in the presence of NS398 (Figure 4d). The addition of $\mathrm{PGE}_{2}$ restored about $75 \%$ of the CMX cells' invasive capacity, strengthening the evidence of the involvement of COX. In addition, the invasive capacity demonstrated by 2.2.15 cells was decreased by NS398 to the levels presented by HepG2 cells (Figure 4d).

We further confirmed our results by using an $\mathrm{HBx}-$ inducible hepatic cell line, $4 \mathrm{pX}$ cells, which express $\mathrm{HBx}$ when tetracycline is removed from the culture 


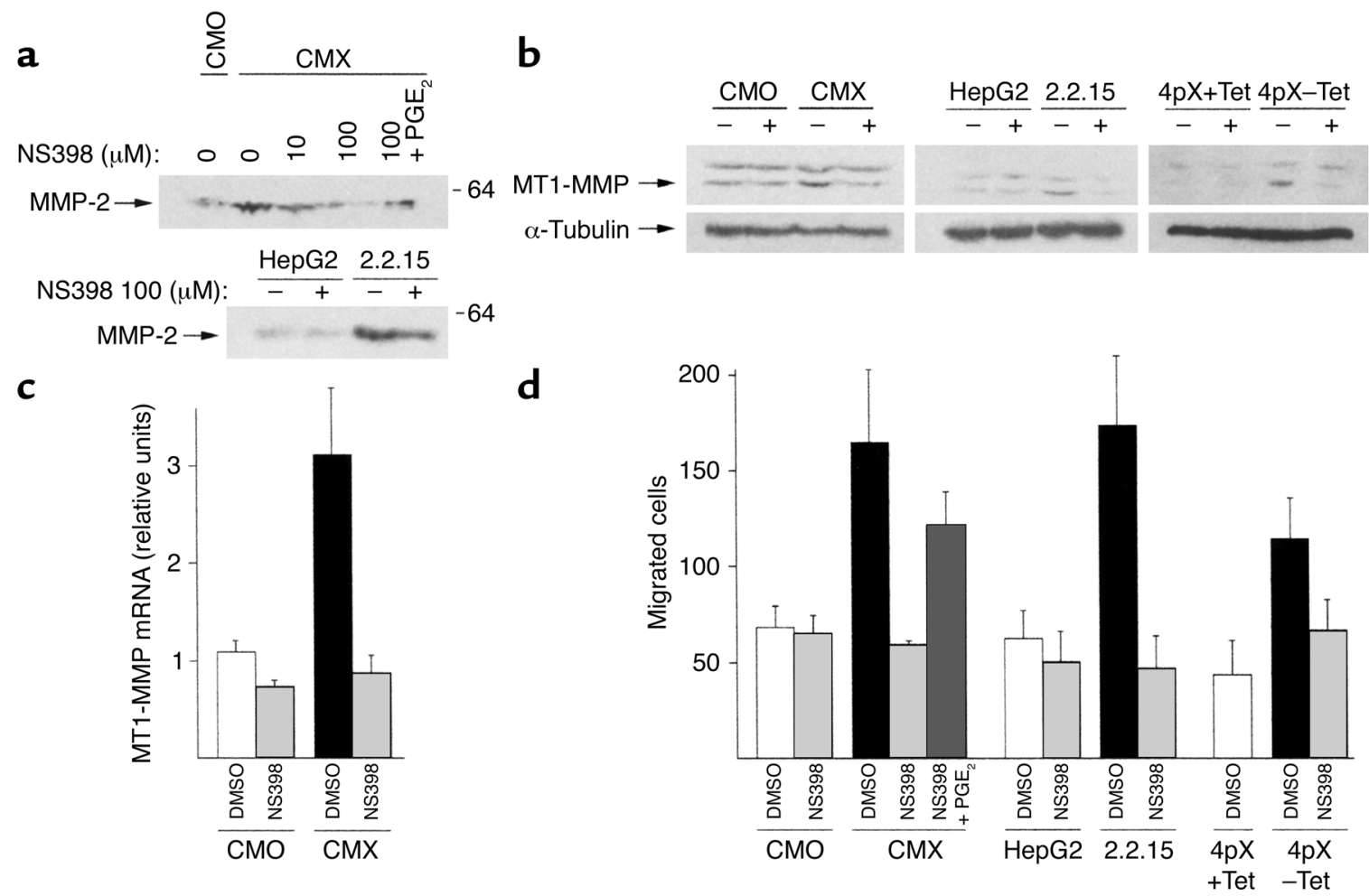

\section{Figure 4}

Induction of MMP-2 activation, MT1-MMP expression, and cell invasion by HBx is sensitive to the COX-2 inhibitor NS398. (a) For 24 hours, CMX cells were grown in the presence of increasing amounts, and HepG2 and 2.2.15 cells were grown in the presence of $100 \mu \mathrm{M}$, of the COX-2-specific inhibitor NS398, along with $10 \mu \mathrm{M} \mathrm{PGE} 2$ where indicated. The presence of activated MMP-2 in the cell lysates was analyzed by Western blot. (b) CMO, CMX, HepG2, and 2.2.15 cells, as well as $4 \mathrm{pX}$ cells in which $\mathrm{HBx}$ expression was induced by removing tetracycline (Tet) for 24 hours, were treated or not treated with $100 \mu \mathrm{M}$ NS398 for 24 hours and lysed, and the amount of MT1-MMP protein was studied by Western blot. (c) CMX and CMO cells were treated or not treated with $100 \mu \mathrm{M}$ NS398 for 24 hours, and the expression of MT1-MMP transcripts was analyzed by quantitative RT-PCR. (d) CMO, CMX, HepG2, 2.2.15, or 4pX cells (with or without tetracycline) were allowed to invade Matrigel-coated Transwells in the presence of $100 \mu \mathrm{M}$ NS398, or DMSO as a control, and $10 \mu \mathrm{M}$ PGE where indicated. Cells that migrated to the lower chamber were quantified as in Figure 1.

medium (21). HBx expression resulted in an upregulation of MT1-MMP as assayed by Western blot (Figure $4 \mathrm{~b}$, right panel). This induction of MT1-MMP expression was prevented by the addition of the COX-2 inhibitor NS398. Moreover, removal of tetracycline resulted in an increased invasive capacity (Figure 4d). Again, inhibition of COX-2 prevented the enhancement of $4 \mathrm{pX}$ cells' invasive capacity.

We then studied the effect of different NSAIDs that preferentially inhibit COX-2, COX-1, or both (meloxicam, indomethacin, and ASA, respectively) on metalloproteinase production and tumor cell invasion induced by HBx. Treatment of CMX cells with meloxicam resulted in both a downregulation of MMP-2 activation and MT1-MMP expression (Figure 5a) and a strong inhibition $(50 \%)$ of their invasive potential (Figure 5b). ASA showed a moderate inhibitory effect on CMX cells, whereas indomethacin had no effect. None of the NSAIDs showed a significant effect on $\mathrm{CMO}$ cell invasion and metalloproteinase production. Together, these results point to COX-2 as a key regulator of the induction of MMP expression and cell invasion by HBx.
HBx induces COX-2 expression. The blockade of cell invasion and MMP expression by COX-2-specific inhibitors prompted us to study whether $\mathrm{HBx}$ was inducing the expression of COX-2. Western blot analysis of COX-2 and COX-1 revealed that CMX, 2.2.15, and 4pX cells without tetracycline expressed higher COX-2 levels than their respective control cells, whereas COX-1 was not detected (Figure 6a). Quantitative RT-PCR analysis of COX-2 confirmed the induction of enzyme in CMX cells and in Chang liver cells transiently transfected with the HBx expression vector $\mathrm{pSV}$-X (Figure $6 \mathrm{~b}$ ). No differences were observed in the transcript levels of mPGES, cPGES, or COX-1 between CMX and CMO cells (data not shown). In addition, we found a twofold increase in the secretion of $\mathrm{PGE}_{2}$ by $\mathrm{HBx}$-bearing cells that was blocked by the addition of $10 \mu \mathrm{M}$ NS398 (not shown). These results demonstrate that $\mathrm{HBx}$ is able to induce $\mathrm{COX}-2$ expression in independent hepatic cell lines.

$H B x$ activates the COX-2 promoter in an NF-AT-dependent manner. To explore the transcriptional regulation of the COX-2 gene by HBx, we transfected Chang liver cells with a luciferase-derived reporter construct bearing the COX-2 promoter (positions -1796 to +104 ), along with 


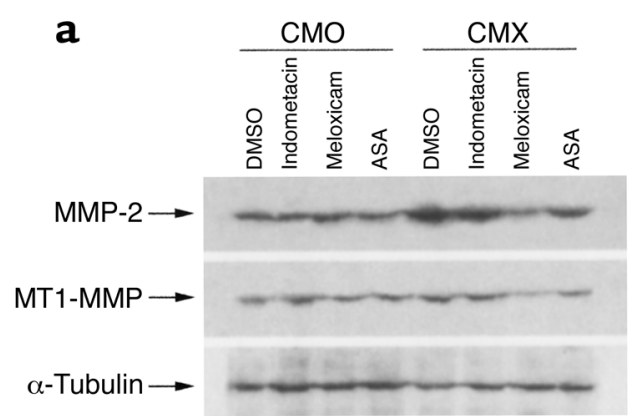

b

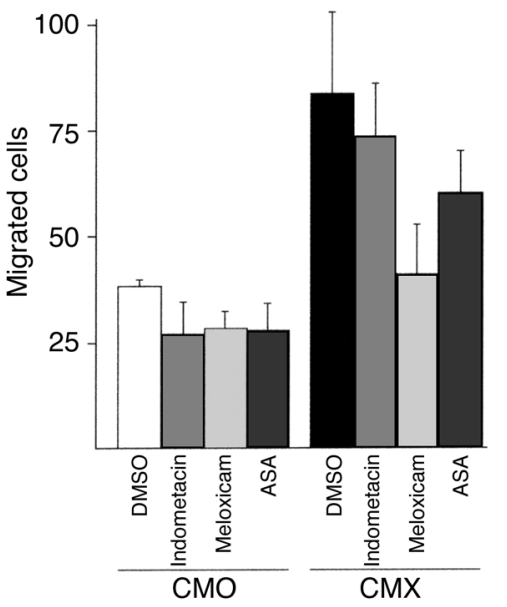

increasing amounts of the $\mathrm{HBx}$-expression vector pSV-X. As shown in Figure 7a, HBx was able to activate the COX-2 promoter in a dose-dependent manner. Similarly, HBx induced the COX-2 promoter when transfected into HepG2 cells (Figure 7a). Deletion of the region spanning positions -1796 to -170 of the COX-2 promoter or point mutation of the proximal NF- $\mathrm{KB}$ site had no effect on the activation of the promoter by HBx. On the contrary, site-directed mutagenesis of the distal and proximal NF-AT sites resulted in a $20 \%$ and a $50 \%$ loss of the $\mathrm{HBx}$-induced promoter activity, respectively (Figure 7b). Moreover, double mutation of both NF-AT sites almost completely abolished the activation of the promoter by HBx.

To further demonstrate the involvement of NF-AT in the $\mathrm{HBx}$-mediated transactivation of the COX-2 promoter, we added a dominant negative NF-AT expression vector to the transfection system, which resulted in a $50 \%$ inhibition of the COX-2 promoter activation by $\mathrm{HBx}$ (Figure 7c). In contrast, transfection of a wildtype NF-AT expression vector showed a strong synergism with $\mathrm{HBx}$ in the activation of the COX-2 promoter. Our results demonstrate that NF-AT, but not $\mathrm{NF}-\kappa \mathrm{B}$, is necessary for the induction of COX-2 by $\mathrm{HBx}$.

\section{Figure 6}

$\mathrm{HBx}$ induces COX-2 expression. (a) The expression of COX-2 and COX-1 was evaluated by Western blot in CMO, CMX, HepG2, 2.2.15, and $4 \mathrm{pX}$ cells (treated or not treated with tetracycline). (b) The presence of COX-2 mRNA was analyzed by quantitative RT-PCR in CMO and CMX cells, and in Chang liver cells transiently transfected with $\mathrm{PSV}$-X or the control vector PSV-hygro.

\section{Figure 5}

Effect of different NSAIDs on MMP-2 activation, MT1-MMP expression, and tumor cell invasion induced by $\mathrm{HBx}$. (a) CMO and CMX cells were grown for 24 hours in the presence of $60 \mu \mathrm{g} / \mathrm{ml}$ indomethacin, $60 \mu \mathrm{g} / \mathrm{ml}$ meloxicam, $600 \mu \mathrm{g} / \mathrm{ml}$ ASA, or DMSO as a control, and the amount of activated MMP-2 and MT1-MMP was analyzed by Western blot. (b) CMX and CMO cells were allowed to invade a Matrigel-coated Transwell in the presence of $60 \mu \mathrm{g} / \mathrm{ml}$ indomethacin, $60 \mu \mathrm{g} / \mathrm{ml}$ meloxicam, $600 \mu \mathrm{g} / \mathrm{ml}$ ASA, or DMSO. Cells that migrated to the lower chamber were quantified as in Figure 1.

\section{Discussion}

Intrahepatic metastasis is a main cause of HCC recurrence and poor prognosis after tumor resection (1). Although chronic infection by HBV is responsible for the development of most HCCs, the specific role of HBV proteins in HCC progression is unknown (1). In this work, we provide evidence, for the first time to our knowledge, that $\mathrm{HBx}$, often the only viral protein expressed by transformed hepatocytes $(2,3)$, induces cell invasion, suggesting its involvement in the metastatic spreading and recurrence of hepatic tumors.

We present both in vivo and in vitro results that support the ability of HBx to induce tumor cell invasion. The chick embryo CAM invasion assay showed that HBx increases the capacity of tumor cells to degrade the ECM, cross the endothelial barrier, and reach the bloodstream. This upregulation of the metastatic abilities of tumor cells was corroborated by the Matrigel invasion assays, in which HBx-expressing cells also displayed increased invasive potential.

Cancer cell invasion requires degradation of the basement membrane, composed mainly of collagen IV,

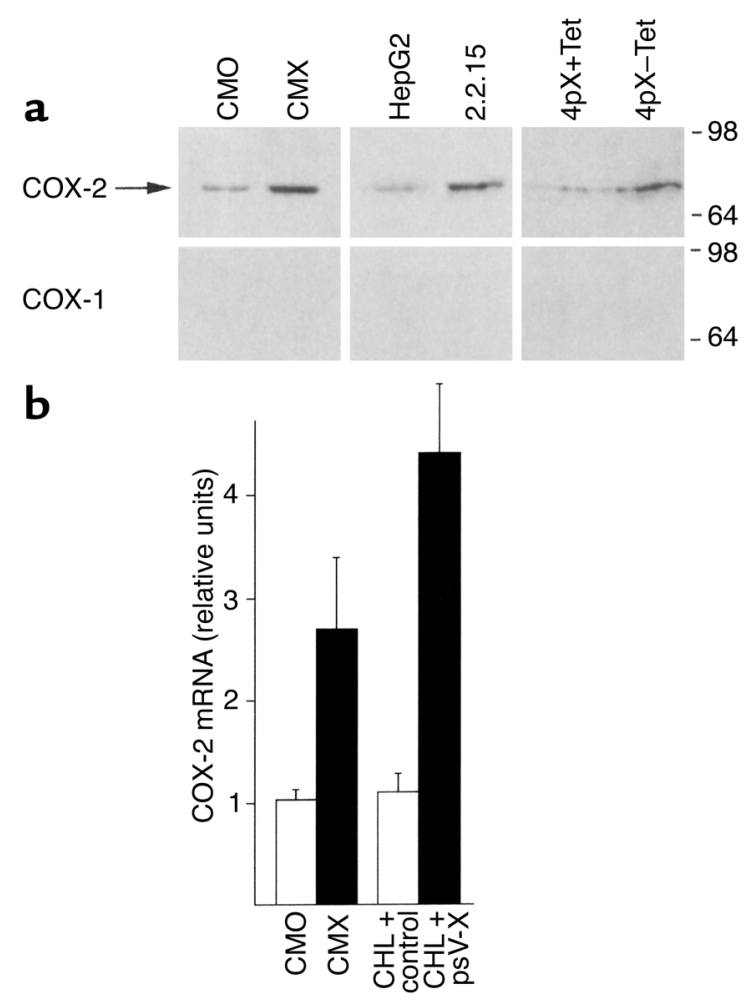




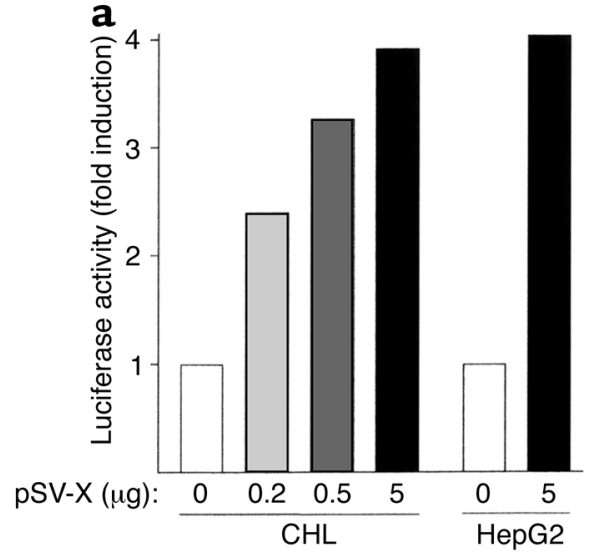

b

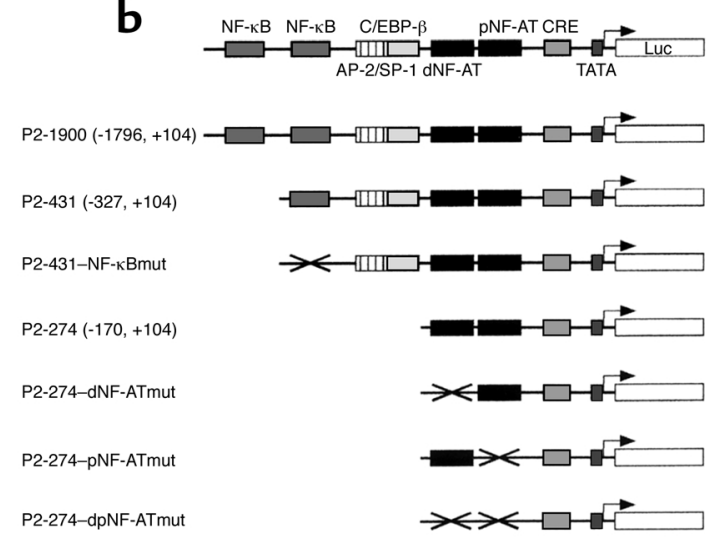

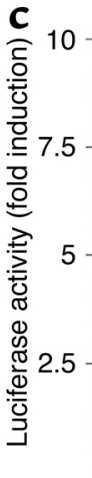

$\mathrm{pSV}-\mathrm{X}$

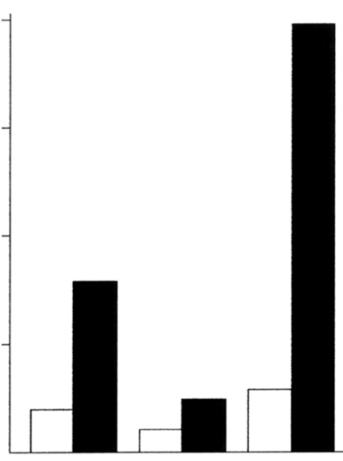

$\overline{\text { Control }} \overline{\text { NF-AT NF-ATwt }}$ dom. neg.

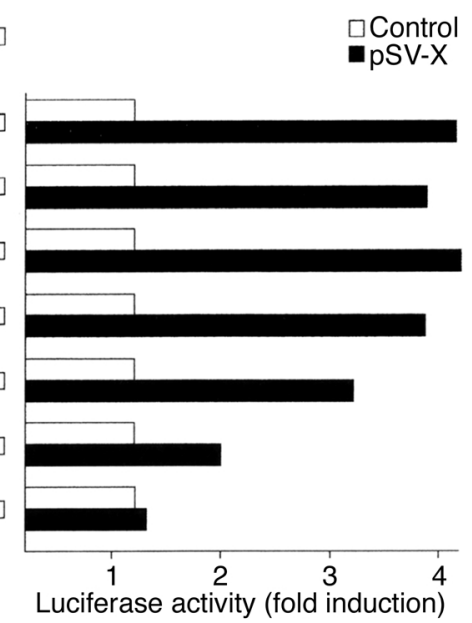

\section{Figure 7}

$\mathrm{HBx}$ induces the COX-2 promoter in an NF-AT-dependent manner. (a) Chang liver and HepG2 cells were transfected with $0.1 \mu \mathrm{g}$ of the luciferase-based COX-2 reporter plasmid P2-1900 (-1796 to +104 ) along with increasing amounts (in Chang liver) or $5 \mu \mathrm{g}$ (in HepG2) of PSV-X. The amount of luciferase was analyzed 18 hours later, and the results were expressed as fold induction over the value without pSV-X. (b) Chang liver cells were transfected as in a with reporter plasmids containing different deletions and point mutations of the COX-2 promoter, along with $5 \mu \mathrm{g}$ of pSV-X or pSV-hygro. The results are expressed as fold induction over the value without PSV-X for each promoter construct and are representative of four independent experiments. (c) Chang liver cells were transfected as in a with $5 \mu \mathrm{g}$ of pSV-X or pSV-hygro and $1 \mu \mathrm{g}$ of the dominant negative NF-AT expression vector pSH102C 4418 , an NF-AT2 wild-type (NF-ATwt) expression vector, or the empty vector $\mathrm{pBJ} 5$ as a negative control. The values are expressed as fold induction over the value with $\mathrm{pBJ} 5$ and without $\mathrm{PSV}-\mathrm{X}$. Results are representative of at least three independent experiments. which is mediated by MMP-2 and MMP-9 (9). Most of the MMP-2 found in the hepatic tumor is produced by the hepatic stellate cells in the surrounding stroma and is secreted as an inactive precursor (11). Although some activated MMP-2 can be found in the fibrotic liver, the highest MMP-2 activation efficiency is found in tumor tissues (11) and correlates with MT1-MMP expression and tumor spreading $(9,10)$. In this regard, MT1-MMP is overexpressed in highly invasive HCCs (10), and activation of MMP-2 is associated with tumor progression and recurrence in HCC patients (11). Moreover, MT1-MMP is also capable of activating other MMPs and directly degrading ECM components and membrane receptors $(9,26)$. This capacity has a pronounced effect on cellular invasiveness independently of MMP-2 processing $(26,27)$. Thus, the induction of MT1-MMP expression by HBx provides the cell with a powerful invasive tool that contributes to tumor spreading and may play a key role in HCC recurrence. NSAIDs show antineoplastic activity in a number of malignancies, due to their ability to inhibit the synthesis of prostaglandins by COX-2 (13). Expression of this enzyme is sufficient to induce tumor growth and invasion by a variety of mechanisms (13), some of which are shared by $\mathrm{HBx}(2)$. In this regard, COX-2 enhances cell migration, expression of MT1-MMP, and MMP-2 activation (12). HBx is also capable of inducing cell motility (17), and we show herein that it upregulates MMP-2 activation and MT1-MMP expression. Both $\mathrm{COX}-2$-dependent invasion and $\mathrm{HBx}$-induced migration are mediated by CD44 $(17,28)$, which is cleaved by MT1-MMP (26). Thus, by regulating COX-2, MT1-MMP, and CD44, HBx may be activating a complex mechanism that will eventually lead to cell invasion.

COX-2-expressing cells induce neovascularization by upregulating different proangiogenic enzymes and growth factors, such as inducible nitric oxide synthase (iNOS), TGF- $\beta$, and VEGF (29). HBx itself induces the expression of iNOS, TGF- $\beta$, and VEGF, and an angiogenic effect of this viral protein has been proposed $(30,31)$. Moreover, COX-2 expression results in increased apoptosis resistance (13), another property shared by HBx in certain systems (8). Thus, the induction of COX-2 expression by $\mathrm{HBx}$ may help to explain the variety of pro-oncogenic effects of this viral protein and may unveil a new target for the treatment of HBV-derived HCC.

Interestingly, NSAID and NS398 treatment of Chang liver and HepG2 cells results in increased response to IFN- $\alpha$, suggesting that COX- 2 expression may result in IFN therapy resistance (32). In this context, the induction of COX-2 by HBx would help the virus to evade the 
immune system, favoring chronic HBV infection and the progression to cirrhosis and HCC.

COX-2 expression can be induced by a number of mitogenic, inflammatory, and pro-oncogenic stimuli (13). It has been recently reported that the calcium-regulated transcription factor NF-AT is essential for the activation of COX-2 transcription by VEGF or PMA plus calcium ionophore (24), whereas activation by LPS or TNF- $\alpha$ is mediated by NF-KB (13). Our results demonstrate that NF-AT, but not NF- $\mathrm{KB}$, is necessary for the induction of COX-2 by $\mathrm{HBx}$. We have previously shown that $\mathrm{HBx}$ activates NF-AT in a calcineurin-dependent manner (25), which is likely due to the increase in intracellular calcium and oxidative stress generated after the interaction of $\mathrm{HBx}$ with the mitochondrion $(33,34)$. These results suggest a role for NF-AT, oxidative stress, and calcium signaling in the induction of inflammatory mediators and tumor development.

In conclusion, our results suggest that the expression of $\mathrm{HBx}$ provides tumor cells with metastatic potential. Since HBx is still expressed after HCC development, we propose that this viral protein may not only have a role in carcinogenesis, as is currently believed, but may also be responsible for tumor recurrence that results from intrahepatic metastasis. The mechanisms described herein by which $\mathrm{HBx}$ induces cell invasion, namely induction of COX-2 and MT1-MMP expression and activity, are in agreement with our previous findings reporting that $\mathrm{HBx}$ induces cell migration, disruption of intercellular adhesion, and cell-matrix interactions and cytoskeleton rearrangements (16-18). The involvement of $\mathrm{HBx}$ in tumor spreading represents a new finding in the contribution of the hepatitis B virus to HCC and provides new clues for understanding the role of $\mathrm{HBx}$ after tumor establishment, unveiling potential new targets in the therapy against this aggressive malignancy.

\section{Acknowledgments}

This work was supported by a grant from the Ministerio de Ciencia y Tecnología (SAF 01/0305 to M. LópezCabrera). E. Lara-Pezzi was supported by a postdoctoral fellowship from the Comunidad Autónoma de Madrid. We are grateful to Arantxa Rosado Díez and Ester Leonardo for technical assistance. We thank Ourania Andrisani for providing us with $4 \mathrm{pX}$ cells, British Biotech for supplying BB-3103, and Innogenetics $\mathrm{GmbH}$ for supplying meloxicam.

1. Schafer, D.F., and Sorrell, M.F. 1999. Hepatocellular carcinoma. Lancet. 353:1253-1257.

2. Feitelson, M.A., and Duan, L.-X. 1997. Hepatitis B virus X antigen in the pathogenesis of chronic infections and the development of hepatocellular carcinoma. Am. J. Pathol. 150:1141-1157.

3. Su, Q., et al. 1998. Expression of hepatitis B virus X protein in HBV-infected human livers and hepatocellular carcinomas. Hepatology. 27:1109-1120.

4. Kim, C.M., et al. 1991. HBx gene of hepatitis B virus induces liver cancer in transgenic mice. Nature. 351:317-320.

5. Slagle, B.L., Lee, T.H., Medina, D., Finegold, M.J., and Butel, J.S. 1996. Increased sensitivity to the hepatocarcinogen diethylnitrosamine in transgenic mice carrying the hepatitis B virus X gene. Mol. Carcinog. 15:261-269.

6. Terradillos, O., et al. 1997. The hepatitis B virus X gene potentiates c-myc-induced liver oncogenesis in transgenic mice. Oncogene. 14:395-404.

7. Kekulé, A., Lauer, U., Weiss, L., Luber, B., and Hofsschneider, P. 1993.
Hepatitis B virus transactivator $\mathrm{HBx}$ uses a tumour promoter signalling pathway. Nature. 361:742-745.

8. Shih, W.L., Kuo, M.L., Chuang, S.E., Cheng, A.L., and Doong, S.L. 2000 Hepatitis B virus X protein inhibits transforming growth factor-betainduced apoptosis through the activation of phosphatidylinositol 3-kinase pathway. J. Biol. Chem. 275:25858-25864.

9. Werb, Z. 1997. ECM and cell surface proteolysis: regulating cellular ecology. Cell. 91:439-442.

10. Harada, T., et al. 1998. Membrane-type matrix metalloproteinase-1 (MT1-MMP) gene is overexpressed in highly invasive hepatocellular carcinoma. J. Hepatol. 28:231-239.

11. Théret, N., et al. 2001. Increased extracellular matrix remodelling is associated with tumor progression in human hepatocellular carcinoma. Hepatology. 34:82-88.

12. Tsujii, M., Kawano, S., and DuBois, R.N. 1997. Cyclooxygenase-2 expression in human colon cancer cells increases metastatic potential. Proc. Natl. Acad. Sci. USA. 94:3336-3340.

13. Williams, C.S., Mann, M., and DuBois, R.N. 1999. The role of cyclooxygenases in inflammation, cancer and development. Oncogene. 18:7908-7916.

14. Koga, H., et al. 1999. Expression of cyclooxygenase- 2 in human hepatocellular carcinoma: relevance to tumor dedifferentiation. Hepatology. 29:688-696.

15. Liu, C.H., et al. 2001. Overexpression of cyclooxygenase- 2 is sufficient to induce tumorigenesis in transgenic mice. J. Biol. Chem. 276:18563-18569.

16. Lara-Pezzi, E., et al. 2001. Effect of the hepatitis B virus HBx protein on integrin-mediated adhesion to and migration on extracellular matrix. J. Hepatol. 34:409-415.

17. Lara-Pezzi, E., et al. 2001. The hepatitis B virus $\mathrm{X}$ protein $(\mathrm{HBx})$ induces a migratory phenotype in a CD44-dependent manner: possible role of $\mathrm{HBx}$ in invasion and metastasis. Hepatology. 33:1270-1281.

18. Lara-Pezzi, E., Roche, S., Andrisani, O.M., Sanchez-Madrid, F., and LopezCabrera, M. 2001. The hepatitis B virus HBx protein induces adherens junction disruption in a src-dependent manner. Oncogene. 20:3323-3331.

19. Lee, J.S., and Thorgeirsson, S.S. 2002. Functional and genomic implications of global gene expression profiles in cell lines from human hepatocellular cancer. Hepatology. 35:1134-1143.

20. Wang, W., London, W.T., Lega, L., and Feitelson, M.A. 1991. HBxAg in the liver from carrier patients with chronic hepatitis and cirrhosis. Hepatology. 14:29-37.

21. Tarn, C., Bilodeau, M.L., Hullinger, R.L., and Andrisani, O.M. 1999. Differential immediate early gene expression in conditional hepatitis $\mathrm{B}$ virus pX-transforming versus nontransforming hepatocyte cell lines. J. Biol. Chem. 274:2327-2336.

22. Gálvez, B.G., Matías-Román, S., Albar,J.P., Sánchez-Madrid, F., and Arroyo, A.G. 2001. Membrane type 1-matrix metalloproteinase is activated during migration of human endothelial cells and modulates endothelial motility and matrix remodeling. J. Biol. Chem. 276:37491-37500.

23. Mira, E., Lacalle, R.A., Gómez-Moutón, C., Leonardo, E., and Mañés, S. 2002. Quantitative determination of tumor cell intravasation in a real-time polymerase chain reaction-based assay. Clin. Exp. Metastasis. 19:313-318.

24. Iñiguez, M.A., Martínez-Martínez, S., Punzón, C., Redondo, J.M., and Fresno, M. 2000. An essential role of the nuclear factor of activated T cells in the regulation of the expression of the cyclooxygenase- 2 gene in human T lymphocytes. J. Biol. Chem. 275:23627-23635.

25. Lara-Pezzi, E., Armesilla, A.L., Majano, P.L., Redondo, J.M., and LopezCabrera, M. 1998. The hepatitis B virus X protein activates nuclear factor of activated T cells (NF-AT) by a cyclosporin A-sensitive pathway. EMBO J. 17:7066-7077.

26. Kajita, M., et al. 2001. Membrane-type 1 matrix metalloproteinase cleaves CD44 and promotes cell migration. J. Cell Biol. 153:893-904.

27. Hotary, K., Allen, E., Punturieri, A., Yana, I., and Weiss, S.J. 2000. Regulation of cell invasion and morphogenesis in a three-dimensional type I collagen matrix by membrane-type matrix metalloproteinases 1,2 and 3. J. Cell Biol. 149:1309-1323.

28. Dohadwala, M., et al. 2001. Non-small cell lung cancer cyclooxygenase-2dependent invasion is mediated by CD44. J. Biol. Chem. 276:20809-20812.

29. Tsujii, M., et al. 1998. Cyclooxygenase regulates angiogenesis induced by colon cancer cells. Cell. 93:705-716.

30. Majano, P.L., et al. 1998. Inducible nitric oxide synthase expression in chronic viral hepatitis. J. Clin. Invest. 101:1343-1352.

31. Yoo, Y.D., et al. 1996. Regulation of transforming growth factor- $\beta 1$ expression by the hepatitis B virus (HBV) X transactivator. J. Clin. Invest. 97:388-395.

32. Giambartolomei, S., et al. 1999. Nonsteroidal anti-inflammatory drug metabolism potentiates interferon alfa signaling by increasing STAT 1 phosphorylation. Hepatology. 30:510-516.

33. Bouchard, M.J., Wang, L.H., and Schneider, R.J. 2001. Calcium signaling by $\mathrm{HBx}$ protein in hepatitis B virus DNA replication. Science. 294:2376-2378.

34. Waris, G., Huh, K.-W., and Siddiqui, A. 2001. Mitochondrially associated hepatitis B virus $X$ protein constitutively activates transcription factors STAT-3 and NF-KB via oxidative stress. Mol. Cell. Biol. 21:7721-7730. 